\title{
L’invalidità civile
}

\author{
Marco Polo ${ }^{1}$, Cosimo Prete ${ }^{2}$, Valerio Cirfera ${ }^{3}$
}

Avvocato, Foro di Lecce

Avvocato, Foro di Lecce

Medico-Chirurgo Dermatologo

\begin{abstract}
This article provides an overview on civil invalidity in Italy, starting from the first law, in 1939, in which the invalid subject was considered and a definition of invalid person was given. During the following years this status has been ascribed to even better defined categories of patients, and now a big system of invalidity calculation, economic and not economic social security benefits has been built. A detailed description of each benefit, including requirements and bureaucratic procedures, follows and a survey on the institutions involved, closes this article
\end{abstract}

Keywords: civil invalidity, handicap, disabled, benefit, allowance, pension

Civil invalidity

Pratica Medica \& Aspetti Legali 2009; 3(3): 107-115

\section{IL RICONOSCIMENTO DELL'INVALIDITÀ CIVILE}

Nel nostro Paese uno dei primi concetti di invalidità è contenuto nella Legge 14 aprile 1939, n. 636 che considera invalido «l'assicurato la cui capacità di guadagno in occupazioni confacenti le sue attitudini sia ridotta in modo permanente per infermità o difetto fisico e mentale, a meno della metà».

Ma è con l'art. 2 della Costituzione ${ }^{1}$ che si afferma solennemente il principio cardine e ineludibile di solidarietà sociale, a cui si è ispirata la legislazione successiva in favore dei disabili, tendente alla liberazione di tali persone dal bisogno, consentendo alle stesse un'esistenza dignitosa ed evitando il rischio di emarginazione.

Lart. 3 della Costituzione, che stabilisce il principio dell'uguaglianza formale e sostanziale, recita:

\footnotetext{
${ }_{1}$ «La Repubblica [...] richiede l'adempimento dei doveri inderogabili di solidarietà politica, economica e sociale»
}

«Tutti i cittadini hanno pari dignità sociale e sono eguali davanti alla legge, senza distinzioni di sesso, di razza, di lingua, di opinioni politiche, di condizioni personali e sociali. È compito della Repubblica rimuovere gli ostacoli che, limitando di fatto la libertà e l'uguaglianza dei cittadini, impediscono il pieno sviluppo della persona umana e l'effettiva partecipazione di tutti i lavoratori all'organizzazione politica, economica e sociale del Paese». In particolare, l'art. 38 della Costituzione dichiara: «Ogni cittadino inabile al lavoro e sprovvisto dei mezzi necessari per vivere ha diritto al mantenimento e all'assistenza sociale».

L'assistenza pubblica, pertanto, nella quale va ricompresa l'invalidità civile, interviene in favore dei cittadini che, sprovvisti di mezzi, per ragioni a loro non ascrivibili, si trovano nelle condizioni di non potersi procurare autonomamente quanto necessario al vivere civile.

Tra i più significativi interventi legislativi corrispondenti al dettame dei summenzionati principi costituzionali si possono annoverare la Legge 5 ottobre 1962, n. 1539 ("Provvedimenti in favo- 
re dei mutilati ed invalidi civili"), che disciplina le assunzioni lavorative dei mutilati e invalidi civili; la Legge 6 agosto 1966, n. 625² ; la Legge 2 aprile 1968 , n. 482 ("Provvidenze a favore dei mutilati ed invalidi civili”), che sancisce il diritto al lavoro dei disabili; la Legge 30 marzo 1971, n. 118 ("Conversione in legge del DL 30 gennaio 1971, n. 5 e nuove norme in favore dei mutilati e invalidi civili”), che prevede il diritto per i disabili di alcuni servizi essenziali (assistenza sanitaria, riabilitazione, scuola, ecc.); la Legge 4 agosto 1977, n. 517 per l'integrazione scolastica; la Legge 5 febbraio 1992 , n. $104^{4}$ ("Legge quadro per l'assistenza, l'integrazione sociale e i diritti delle persone handicappate"), che, dettando i principi dell'ordinamento in materia di diritti, integrazione sociale e assistenza delle persone disabili, comprende praticamente tutti gli aspetti della loro vita quotidiana, anche se le provvidenze economiche sono disciplinate da altri provvedimenti normativi.

Va però precisato che sostanzialmente le infermità fisiche, psichiche e sensoriali consentono l'accesso ai benefici previsti dalla Legge n. 104/1992 nei soli casi in cui comportino una condizione di svantaggio sociale o di emarginazione.

Notevole importanza rivestono altresì il trasferimento all'INPS di tutte le competenze relative all'erogazione di benefici economici agli invalidi civili, con conferimento alle Regioni delle funzioni di concessione [1], nonché l'estensione della fruizione delle provvidenze e delle prestazioni, anche economiche, di assistenza sociale agli stranieri titolari della carta di soggiorno o di permesso di soggiorno non inferiore a un anno [2].

È stata poi prevista la promozione dell'inserimento e dell'integrazione lavorativa delle persone di-

${ }^{2}$ Art. 1: «Il Ministero della Sanità provvede all'assistenza sanitaria specifica diretta al recupero funzionale dei mutilati ed invalidi civili appartenenti alla categoria dei motulesi e dei neurolesi che versano in istato di bisogno e la cui invalidità possa essere ridotta mediante idoneo trattamento di riabilitazione»

${ }^{3}$ Art. 2, comma II: "La scuola attua forme di integrazione a favore degli alunni portatori di handicaps con la prestazione di insegnanti specializzati assegnati ai sensi dell'articolo 9 del decreto del Presidente della Repubblica 31 ottobre 1975, n. 870» Art. 2, comma III: «Devono inoltre essere assicurati la necessaria integrazione specialistica, il servizio socio-psicopedagogico e forme particolari di sostegno secondo le rispettive competenze dello Stato e degli enti locali preposti, nei limiti delle relative disponibilità di bilancio e sulla base del programma predisposto dal consiglio scolastico distrettuale»

${ }^{4}$ L'art. 3 di tale legge definisce persona handicappata «colui che presenta una minorazione fisica, psichica o sensoriale stabilizzata o progressiva che è causa di difficoltà di apprendimento, di relazione o di integrazione lavorativa, e tale da determinare un processo di svantaggio sociale o di emarginazione» sabili, nel cui ampio ambito sono da ricomprendersi tutte le categorie di invalidi, civili, del lavoro o per malattia professionale, di guerra, di servizio, non vedenti e sordomuti, attraverso servizi di sostegno e di collocamento mirato [3].

In tema di invalidità civile, fondamentale è la Legge 8 novembre 2000 , n. 328 ("Legge quadro per la realizzazione del sistema integrato di interventi e servizi sociali”) il cui art. 1, comma I, prevede: «La Repubblica assicura alle persone e alle famiglie un sistema integrato di interventi e servizi sociali, promuove interventi per garantire la qualità della vita, pari opportunità, non discriminazione e diritti di cittadinanza, previene, elimina o riduce le condizioni di disabilità, di bisogno e di disagio individuale e familiare, derivante $[. .$.$] da inadegua-$ tezza di reddito, difficoltà sociali e condizioni di non autonomia, in coerenza degli articoli 2, 3 e 38 della Costituzione».

Negli ultimi anni, in un'ottica antidiscriminatoria dei soggetti affetti da infermità e tendente fondamentalmente all'eliminazione delle barriere sociali ancora esistenti, a partire da quelle terminologiche, nonché alla valorizzazione degli stessi soggetti, al concetto di disabile si è sostituito quello di "diversamente abile".

Premesso l'iter dei principali interventi normativi in materia di invalidità civile, specificamente si evidenzia che una prima definizione normativa dell'invalidità civile è contenuta nell'art. 2 della Legge $\mathbf{n}$. 118/1971, che considera mutilati e invalidi civili «i cittadini affetti da minorazioni congenite o acquisite, anche a carattere progressivo, compresi gli irregolari psichici per oligofrenie di carattere organico o dismetabolico, insufficienze mentali derivanti da difetti sensoriali e funzionali che abbiano subito una riduzione permanente della capacità lavorativa non inferiore ad un terzo o, se minori di anni 18 , che abbiano difficoltà persistenti a svolgere $\mathrm{i}$ compiti e le funzioni proprie della loro età».

Diversamente da quanto previsto dall'art. 5 della Legge 6 agosto 1966, n. $625^{5}$, che escludeva il malato psichico puro dalla categoria degli aventi diritto, dunque, nel vigente concetto di invalido civile rientrano anche i soggetti affetti da insufficienze mentali derivanti da minorazioni puramente psichiche. I minorati psichici rientrano pertanto nell'ampia categoria degli affetti da menomazioni congenite o acquisite di cui all'art. 2 della Legge n. 118/1971.

L'art. 2 della Legge 118/1971, originariamente, era stato interpretato in senso restrittivo, in modo

\footnotetext{
${ }^{5}$ «Agli invalidi civili di età superiore agli anni 18 nei cui confronti sia accertata una totale e permanente inabilità lavorativa non di natura psichica, che versino in stato di bisogno e non fruiscano di pensioni, assegni o rendite di qualsiasi natura o provenienza, è concesso, a carico dello Stato ed a cura del Ministero dell'Interno, un assegno mensile di assistenza nella misura di lire ottomila»
} 
tale da escludere dal riconoscimento i soggetti affetti da malattie psichiche cosiddette pure, primarie o essenziali, come gli schizofrenici e tutti coloro la cui minorazione non fosse di carattere organico.

Negli anni successivi, interpretando lo spirito della legge, la giurisprudenza ha riconosciuto che le malattie psichiche, di ogni natura, rientrano pienamente nella categoria delle menomazioni congenite o acquisite che possono comportare il riconoscimento dell'invalidità civile [4].

Secondo l'art. 1 della Legge n. 509/1988, «Le minorazioni congenite od acquisite di cui all'art. $2,2^{\circ}$ comma della L. 30 marzo 1971, n. 118 comprendono gli esiti permanenti delle infermità fisiche e/o psichiche e sensoriali che comportano un danno funzionale permanente».

Inoltre, l'art. 6 del D. Lgs. 23 novembre 1988, n. 509, ha aggiunto all'art. 2 della Legge n. 118/1971 la seguente disposizione: «Ai soli fini dell'assistenza socio-sanitaria e della concessione dell'indennità di accompagnamento si considerano mutilati ed invalidi i soggetti ultrasessantacinquenni che abbiano difficoltà persistenti a svolgere i compiti e le funzioni proprie della loro età».

La ratio del sopra citato intervento normativo è dunque ravvisabile nella protezione delle persone anziane che, unitamente ai minori, sono i soggetti più deboli, privi di qualsiasi altra tutela.

I compiti e le funzioni proprie dell'età, pertanto, presuppongono una integrità psico-fisica; la riduzione di tale integrità come requisito fondamentale per accedere ai benefici assistenziali non viene quantificata dalla suddetta normativa. Affinché l'anziano possa acquisire lo status di invalido civile è quindi sufficiente che lo stesso abbia difficoltà persistenti a svolgere i compiti e le funzioni proprie della sua età.

Il soggetto ultrasessantacinquenne riconosciuto invalido civile usufruisce di prestazioni esclusivamente assistenziali e non ha diritto a percepire l'assegno di invalidità o la pensione di inabilità; può invece usufruire della pensione sociale, qualora ne sussistano le condizioni.

Ai sensi dell'ultimo comma dell'art. 2 della Legge n. 118/1971, "Sono esclusi gli invalidi per cause di guerra, di lavoro, di servizio, nonché i ciechi e i sordomuti per i quali provvedono altre leggi», in quanto per queste categorie l'invalidità deriva da una causa specifica (la guerra o le prestazioni lavorative).

In particolare, secondo l'art. 7 della Legge 10 febbraio 1962 , n. 66, cieco è da intendersi «ogni cittadino affetto da cecità congenita o contratta in seguito a cause che non siano di guerra, infortunio sul lavoro o di servizio». A tal proposito si rileva che tale legge, integrata dalla successiva Legge 27 maggio 1970, n. 382, attualmente in vigore, è stato il primo intervento legislativo a tutelare una forma di invalidità quale la cecità attraverso l'istituzione di una pensione.

Sordomuto $^{6}$ viene invece definito «il minorato sensoriale dell'udito affetto da sordità congenita o acquistata durante l'età evolutiva che gli abbia impedito il normale apprendimento del linguaggio parlato, purché la sordità non sia di natura esclusivamente psichica o dipendente da causa di guerra, di servizio, di lavoro» (art. 1 della Legge n. 381/1970, istitutiva della pensione a favore dei sordomuti).

Inoltre, ai sensi dell'art. 3, comma I, della sopra citata Legge n. 104/1992, è considerata persona handicappata "colui che presenta una minorazione fisica, psichica o sensoriale, stabilizzata o progressiva, che è causa di difficoltà di apprendimento, di relazione o di integrazione lavorativa e tale da determinare un processo di svantaggio sociale o di emarginazione».

Ai fini di poter usufruire delle provvidenze previste da detta legge, è necessario che la condizione soggettiva di persona handicappata si identifichi con la menomazione delle condizioni di vita del soggetto, causata dalla compresenza di patologie e di altri fattori, e non con lo stato invalidante che ne è all'origine e che costituisce, invece, il titolo per fruire della corresponsione delle prestazioni assistenziali e previdenziali, come la pensione di invalidità e l'indennità di accompagnamento [5].

Lart. 39 della Legge 6 marzo 1998, n. 40, infine, stabilisce che gli stranieri titolari della carta di soggiorno o di permesso di soggiorno di durata non inferiore a un anno e i minori iscritti nella loro carta, sono equiparati ai cittadini italiani ai fini della fruizione delle prestazioni, anche economiche, di assistenza sociale previste per gli invalidi civili.

Inoltre l'art. 80, comma XIX, della Legge 23 dicembre 2000, n. 388 (cosiddetta "Legge Finanziaria 2001"), ha stabilito che «l'assegno sociale e le provvidenze economiche che costituiscono diritti soggettivi in base alla legislazione vigente in materia di servizi sociali sono concessi, alle condizioni previste dalla legislazione medesima, agli stranieri che siano titolari di carta di soggiorno; per le altre prestazioni e servizi sociali l'equiparazione con i cittadini italiani è consentita a favore degli stranieri che siano almeno titolari di permesso di soggiorno di durata non inferiore ad un anno».

Dopo avere definito le categorie di soggetti a cui è attribuibile lo status di invalido civile sulla base delle condizioni di salute, passiamo ora ad analizzare i requisiti stabiliti dal legislatore per la concessione delle prestazioni in favore degli stessi. Innanzitutto è necessaria la coesistenza del possesso della cittadinanza italiana e della residenza.

\footnotetext{
${ }^{6}$ Per effetto dell'art. 1 della Legge n. 95/2006, il termine "sordo" sostituisce in tutte le disposizioni legislative il tradizionale termine "sordomuto"
} 
Conseguentemente, un cittadino italiano non può beneficiare di tali prestazioni se risiede all'estero, mentre le medesime possono essere riconosciute allo straniero che si trovi nel territorio italiano, secondo quanto sopra evidenziato.

Deve inoltre riscontrarsi una riduzione permanente della capacità lavorativa, che il Ministero della Sanità, nella Circolare n. 10 del 10/04/1981, definisce "una generica validità fisico-psichica del soggetto", specificando che detta capacità lavorativa «non presuppone peraltro che il soggetto debba necessariamente impiegare la propria efficienza in concreto, ma che possieda la stessa, come fatto meramente potenziale e come parametro medio".

Con l'accertamento del grado di invalidità civile, misurato in valori percentuali, dunque, si valuta l'incidenza della menomazione sulla capacità lavorativa del soggetto. Detta valutazione viene utilizzata solo per le persone di età compresa tra i 18 e i 65 anni (popolazione lavorativa). Per i minori e gli ultrasessantacinquenni si verifica l'effettiva presenza di difficoltà persistenti a svolgere i compiti e le funzioni proprie della loro età.

La valutazione sanitaria, pertanto, è diretta all'accertamento della menomazione e della sua misura, secondo le percentuali di invalidità previste dal Decreto del Ministero della Salute 5 febbraio 1992 ("Approvazione della nuova tabella indicativa delle percentuali d'invalidità per le minorazioni e malattie invalidanti”), elaborate in base alla classificazione internazionale della menomazioni proposta dall'Organizzazione Mondiale della Sanità, con conseguente individuazione dei riflessi negativi sulla capacità lavorativa. Ad ogni menomazione corrisponde pertanto una riduzione della capacità lavorativa.

Secondo quanto disposto dall'art. 1, comma II, del D. Lgs. n. 509/1988, "Ai fini della valutazione della riduzione della capacità lavorativa, le infermità devono essere accertate da apposite indagini cliniche, strumentali e di laboratorio, allo scopo di determinare l'entità delle conseguenze e delle complicanze anatomo-funzionali permanenti ed invalidanti in atto»; il comma IV del medesimo articolo, inoltre, precisa: «La determinazione della percentuale di riduzione della capacità lavorativa deve basarsi: a) sull'entità della perdita anatomica o funzionale, totale o parziale di organi o di apparati; b) sulla possibilità o meno dell'applicazione di apparecchi protesici che garantiscono in modo totale o parziale il ripristino funzionale degli organi o apparati lesi; c) sull'importanza che riveste, in attività lavorative, l'organo o l'apparato sede del danno anatomico o funzionale».

Va anche rilevato che le menomazioni invalidanti devono necessariamente rivestire il carattere della permanenza e non quello della transitorietà. A tal proposito esistono gli istituti della revisione e della verifica.

\section{LE PROVVIDENZE}

Il riconoscimento dei vari gradi di invalidità civile può dare diritto a differenti provvidenze, alcune di natura economica, altre di natura non economica, ma, giova precisarlo, tutte di carattere solo assistenziale e indipendente da qualsiasi versamento contributivo.

Secondo le vigenti previsioni normative in materia di invalidità civile, appartengono alla categoria delle prestazioni assistenziali non economiche:

- prestazioni protesiche e ortopediche (ai sensi della Legge n. 118/1971 e del DM Sanità 20 dicembre 1988 , in presenza di una invalidità superiore a $1 / 3$ o di difficoltà persistenti a svolgere le funzioni proprie dell'età);

- collocamento obbligatorio (ai sensi n. 68/1999 e della Circ. Min. Lavoro n. 4/2000, in presenza di un'invalidità superiore o uguale al $46 \%$ );

- esenzione dal ticket sulla spesa sanitaria (ai sensi della Legge n. 724/1994 e succ., in presenza di un'invalidità superiore o uguale al $67 \%$ ).

Appartengono invece alla categoria delle prestazioni assistenziali economiche:

- assegno mensile di invalidità civile (in presenza di invalidità superiore o uguale al 74\%);

- pensione di inabilità (in presenza di invalidità uguale al $100 \%$ );

- indennità mensile di frequenza per i minori invalidi;

- indennità di accompagnamento;

- pensione sociale e assegno sociale.

Tali prestazioni sono erogate esclusivamente quando il cittadino possiede i requisiti sanitari e socio-economici previsti dalla legge. Va anche precisato che, al compimento del sessantacinquesimo anno di età, la prestazione economica percepita dalla persona già riconosciuta invalida civile o sordomuta, si convertirà automaticamente in pensione sociale.

La natura assistenziale delle prestazioni economiche è indipendente da qualsiasi versamento contributivo del soggetto: si considera, dunque, esclusivamente la precaria situazione reddituale come elemento costitutivo del diritto e non la mera condizione di erogabilità.

È considerevole la differenza (e l'incompatibilità) esistente tra le prestazioni economiche assistenziali e quelle previdenziali (ad esempio, l'assegno ordinario di invalidità e la pensione ordinaria di inabilità), per beneficiare delle quali è necessario fare riferimento ai requisiti contributivi e alla posizione lavorativa della persona. 
L'assegno mensile di invalidità civile, istituito dall' art. 13 della Legge n. 118/1971 ed erogato in 13 mensilità, è concesso agli invalidi civili di età compresa tra i 18 e i 65 anni, con una percentuale di invalidità non inferiore al $74 \%$ (invalidità parziale, art. 9 del D. Lgs. n. 509/1988), che versino in stato di bisogno economico (requisito reddituale, art. 12, comma 3, della Legge n. 412/1991) e che risultino incollocati al lavoro (art. 13, comma 2, della Legge n. 118/1971), secondo quanto risulta dal certificato di iscrizione nelle liste di collocamento o presentazione della relativa domanda, ma il soggetto non deve trovarsi in uno stato di disoccupazione per avere rifiutato un lavoro adatto alle proprie condizioni fisiche.

I requisiti summenzionati devono coesistere tutti e necessariamente ai fini della concessione di tale prestazione economica.

Occorre tuttavia evidenziare che, nonostante la previsione normativa, gli invalidi civili che svolgono un'attività lavorativa possono usufruire dell'assegno mensile a condizione che non superino il limiti reddituali previsti ex lege, e, al compimento dell'età pensionabile, l'assegno viene trasformato in pensione di vecchiaia.

Tale provvidenza econonomica non ha carattere definitivo: vale, infatti, fino a un massimo di tre anni ed è rinnovabile su domanda del beneficiario, che viene pertanto sottoposto a una nuova visita medico-legale.

Lassegno mensile di invalidità civile viene concesso dal primo giorno del mese successivo a quello di presentazione della domanda, salvo diversa disposizione della commissione medica di accertamento, ed è incompatibile con gli altri trattamenti economici.

Inoltre le condizioni di salute possono subire variazioni nel corso degli anni. In tali casi è necessario richiedere un nuovo accertamento di invalidità, specificando, qualora si sia verificato un peggioramento delle condizioni medesime, che trattasi di aggravamento: se riconosciuto, può comportare una modifica del beneficio economico erogato.

Verificatasi la morte del titolare della provvidenza, gli eredi devono quanto prima darne comunicazione all'ente erogatore; tuttavia gli stessi hanno diritto a ricevere i ratei maturati e non ancora riscossi.

La pensione di inabilità civile, istituita con l'art. 12 della Legge n. 118/1971 ed erogata in 13 mensilità, con decorrenza dal primo giorno del mese successivo a quello di presentazione della domanda per l'accertamento dell'inabilità, può essere concessa agli invalidi civili di età compresa tra i 18 e i 65 anni, con una totale e permanente inabilità lavorativa (invalidità totale).

Tale pensione, dunque, si differenzia dall'assegno mensile di invalidità civile poiché, pur essendo anch'essa una prestazione con lo scopo di com- pensare la mancanza di reddito personale, è erogata a persone con una percentuale di invalidità pari al $100 \%$ e non soltanto pari o superiore al $74 \%$. La somma erogata è pari a quella dell'assegno di invalidità.

Lindennità mensile di frequenza per i minori invalidi, prevista dall'art. 1 della Legge 11 ottobre 1990, n. 289, e di importo pari all'assegno mensile di invalidità, è riconosciuta ai minorenni che abbiano difficoltà persistenti a svolgere i compiti e le funzioni della propria età oppure che siano ipoacusici, sempre che non superino i limiti reddituali tassativamente previsti dalla legge.

La concessione dell'indennità è subordinata alla frequenza continua o periodica di centri ambulatoriali o di centri diurni, anche di tipo semiresidenziale, pubblici o privati, operanti in regime convenzionale, specializzati nel trattamento terapeutico o nella riabilitazione e nel recupero di persone portatrici di handicap (art. 1, comma II, della Legge n. 289/1990), ovvero alla frequenza continua o periodica di scuole pubbliche o private di ogni ordine e grado, centri di formazione o di addestramento professionale finalizzati al reinserimento sociale (art. 1, comma III, della Legge 289/1990).

Il minorenne riconosciuto invalido civile o sordomuto che percepisce l'indennità di frequenza, un mese prima di compiere il diciottesimo anno, deve presentare domanda di riconoscimento di invalidità civile per sottoporsi a visita medica, a seguito della quale gli verrà rilasciato un verbale con l'indicazione della percentuale di invalidità accertata ed eventuali provvidenze economiche.

L'indennità di accompagnamento è prevista dall'art. 1 della Legge 11 febbraio 1980 , n. 18, ("Indennità di accompagnamento agli invalidi civili totalmente inabili”) e può essere concessa ai «mutilati ed invalidi civili totalmente inabili per affezioni fisiche o psichiche $[\ldots]$ che si trovano nell'impossibilità di deambulare senza l'aiuto permanente di un accompagnatore o, non essendo in grado di compiere gli atti quotidiani della vita, abbisognano di assistenza continua».

Tale indennità è prevista al solo titolo della minorazione, cioè a prescindere dal reddito del soggetto $^{7}$, e senza tenere in considerazione l'età. Pertanto possono percepirla sia i minorenni che gli ultrasessantacinquenni.

La ratio dell'indennità di accompagnamento, quindi, consiste nell'incoraggiare le famiglie a tenere presso di sé le persone invalide, evitandone il ricovero e la conseguente emarginazione, è giustificata anche nei confronti delle famiglie non povere e contribuisce a sollevare lo Stato da un onere che sarebbe ben più gravoso [6].

\footnotetext{
${ }^{7}$ In tal senso: Cass. Civ. Sez. Unite, n. 11843/1992; Cass. Civ., n. 4518/1993, n. 7917/1995, n. 11295/2000, n. $1268 / 2005$
} 
Il diritto all'indennità, pertanto, non può essere concesso a favore di soggetti ricoverati gratuitamente nelle strutture pubbliche o private che percepiscono rette a carico dello Stato.

Inoltre la persona ultrasessantacinquenne, già riconosciuta parzialmente invalida, può presentare domanda di aggravamento al fine di ottenere l'indennità di accompagnamento.

La pensione sociale e l'assegno sociale, disciplinati dall'art. 19 della Legge n. 118/1971, sono istituti assistenziali introdotti in favore di cittadini che abbiano compiuto 65 anni e si trovino nelle condizioni reddituali previste. Tali trattamenti economici sostituiscono l'assegno mensile e la pensione di inabilità erogati agli invalidi civili che abbiano raggiunto il sessantacinquesimo anno di età.

Nel caso in cui l'importo della pensione o dell'assegno sociale risulti inferiore a quello della pensione di inabilità o dell'assegno mensile di cui l'invalido civile era titolare, la differenza viene corrisposta con un assegno.

L'accertamento dell'invalidità è necessario ai fini della concessione dei benefici e delle provvidenze economiche previsti dal sistema normativo vigente. Pertanto il cittadino che ritenga di essere in possesso dei requisiti richiesti deve presentare domanda presso la ASL di residenza, redatta in carta semplice e corredata della certificazione medica da cui risulti la natura dell'infermità.

Ai sensi dell'art. 1 della Legge 15 ottobre 1990 , n. 295, infatti, "gli accertamenti sanitari relativi alle domande per ottenere la pensione, l'assegno o le indennità di invalidità civile $[. .$.$] nonché gli$ accertamenti sanitari relativi alle domande per usufruire di benefici diversi da quelli dinanzi indicati sono effettuati dalle Unità Sanitarie Locali», al cui interno operano una o più commissioni mediche incaricate, competenti anche per l'accertamento della sussistenza della situazione di handicap, secondo quanto previsto dalla Legge n. 104/1992.

Qualora si tratta di domanda diretta all'ottenimento dell'indennità di accompagnamento, il certificato medico, oltre a definire la malattia invalidante, deve contenere la dicitura di «persona impossibilitata a deambulare senza l'aiuto permanente di un accompagnatore o persona che necessita di assistenza continua non essendo in grado di compiere gli atti quotidiani della vita».

Con la stessa domanda e con un unico accertamento sanitario è possibile ottenere sia la certificazione di invalidità civile, cecità o sordomutismo, che quella di handicap.

Le suddette commissioni sono composte da un medico specialista in medicina legale, cui spetta la presidenza, e da altri due medici, di cui uno deve essere scelto tra gli specialisti in medicina del lavoro (art. 1, comma II, della Legge n. 295/1990), integrate, di volta in volta, con un sanitario in rappresentanza delle associazioni di categoria.
Gli accertamenti relativi allo stato di handicap sono effettuati dalla medesima commissione che accerta l'invalidità civile, integrata da un operatore sociale, e da un esperto nei casi da esaminare, secondo l'art. 4 della Legge n. 104/1992.

Le commissioni mediche periferiche per le pensioni di guerra del Ministero dell'Economia e delle Finanze, a partire dal 1988, hanno assunto la denominazione di commissioni mediche periferiche per le pensioni di guerra e di invalidità civile, con funzioni di commissioni di verifica (art. 3 DL n. 173/1988), e aventi funzioni di controllo dei verbali di visita redatti dalle commissioni mediche operanti presso le ASL. Dette commissioni sono composte da ufficiali medici del servizio permanente o delle categorie in congedo, da medici appartenenti al personale civile dello Stato e da altri membri indicati nell'art. 205 del DPR n. 915/1978 e dal DM $387 / 1991$. Anch'esse sono integrate da un medico in rappresentanza delle associazioni di categoria. Secondo quanto previsto dall'art. 1, comma VII, della Legge n. 295/1990, appena effettuata la visita, la ASL trasmette il verbale alla commissione medica periferica per le pensioni di guerra e di invalidità civile, salvi i casi di giudizio negativo sull'esistenza di invalidità, o con infermità valutata in misura inferiore al $74 \%$.

La ASL, entro 3 mesi dalla presentazione della domanda, deve convocare il richiedente per la visita di accertamento dello stato d'invalidità.

Se la visita non viene fissata entro tale termine, l'interessato può presentare una diffida a provvedere all'Assessorato alla Sanità della Regione territorialmente competente che fissa la data della visita, da effettuarsi da parte della commissione operante presso la ASL di appartenenza, entro il termine complessivo di 9 mesi dalla data di presentazione della domanda, ovvero, se la diffida sia presentata oltre il sesto mese dalla domanda, non oltre 90 giorni dalla sua presentazione, dandone formale comunicazione all'interessato (art. 3, comma I, DPR 698/1994).

Nell'ipotesi in cui il soggetto sia ricoverato o domiciliato in una ASL diversa da quella di effettiva residenza, si può richiedere l'accertamento in rogatoria.

Qualora l'interessato non si presenti a visita, viene convocato per una seconda volta entro i successivi tre mesi ma, in caso di una nuova mancata presentazione, la domanda di riconoscimento dell'invalidità perde efficacia ma potrà essere ripresentata (art. 5, comma I, DM 387/1991).

Se il soggetto convocato a visita è impossibilitato a presentarsi presso la commissione medica, può richiedere la visita domiciliare motivando l'impedimento con idonea documentazione sanitaria e indicando la data in cui detta visita dovrebbe essere effettuata (art. 1, comma VII, del DPR n. 688/1994). 
Durante la visita, il richiedente può farsi assistere da un proprio medico di fiducia (art. 1, comma III, della Legge n. 295/1990) e la commissione può richiedere accertamenti clinici specialistici ulteriori.

Una volta terminati gli accertamenti sanitari, la commissione redige il verbale di visita, da cui deve risultare la riduzione della capacità lavorativa relativa alle singole infermità e quella complessiva.

Nel caso in cui la commissione dell'ASL giudichi che la minorazione accertata sia suscettibile di modificazioni nel corso del tempo, nel verbale deve essere indicato il termine alla scadenza del quale l'interessato deve essere sottoposto a nuova visita (cosiddetta di revisione).

Successivamente alla visita, la commissione dell'ASL trasmette l'esito alla commissione di verifica dalla quale può essere o meno convalidato. Tale commissione può convocare il richiedente per approfondimenti e, durante la visita, può richiedere accertamenti clinici specialistici ulteriori; inoltre può chiedere chiarimenti alla commissione ASL.

Comunque, entro il termine di 60 giorni, la commissione di verifica può richiedere la sospensione della procedura e, in caso contrario, vige il principio del silenzio assenso.

Pertanto l'ASL trasmette all'interessato il verbale contenente l'esito della visita, unitamente alle modalità e ai termini per l'eventuale impugnazione del medesimo.

Se alla visita di revisione l'interessato non si presenta senza giustificato motivo, o non si sottopone ad accertamenti specialistici, viene disposta la sospensione dei pagamenti. Inoltre, qualora l'interessato non presenti adeguata documentazione medica entro 90 giorni dall'avvenuta sospensione, ovvero la documentazione prodotta non sia ritenuta idonea a giustificare la mancata presentazione a visita, viene disposta la revoca della prestazione.

Liter burocratico diretto a ottenere il riconoscimento dell'invalidità, che consta di tre fasi (accertamento, concessione della provvidenza ed erogazione), deve concludersi entro 9 mesi dalla presentazione della domanda (art. 3 del DPR $\mathbf{n}$. 688/1994).

Liter amministrativo della concessione si conclude con una comunicazione del verbale all'interessato, una successiva verifica dei presupposti reddituali tramite un modulo che viene inviato dal Comune di residenza e, infine, con la trasmissione degli atti all'INPS affinché provveda al pagamento della provvidenza economica e degli arretrati.

I benefici economici decorrono dal primo giorno del mese successivo alla data della presentazione della domanda; tuttavia una diversa e successiva data può essere indicata dalla commissione medica.
Sopravvenendo il decesso del richiedente, la commissione dell'ASL può, su formale istanza degli eredi, procedere all'accertamento sanitario esclusivamente in presenza di documentazione medica rilasciata da strutture pubbliche o convenzionate in data antecedente al decesso e "comprovanti, in modo certo, l'esistenza delle infermità e tali da conseguire la formulazione di una esatta diagnosi ed un compiuto e motivato giudizio medico-legale» ${ }^{8}$. Il verbale di visita redatto dalla commissione medica dell'ASL diventa definitivo se la commissione di verifica non provvede alla sospensione della procedura nel citato termine di 60 giorni ovvero se nello stesso termine comunichi di non avere nulla da osservare. Pertanto copia del verbale di visita viene inviata all'interessato dalla commissione medica dell'ASL.

Nel caso in cui la percentuale di invalidità riconosciuta comporti il diritto dell'interessato a una provvidenza economica, altra copia autentica del verbale viene trasmessa agli enti competenti per gli ulteriori adempimenti.

Relativamente all'impugnazione dei provvedimenti in materia di invalidità civile, va evidenziato che, dopo la soppressione del ricorso amministrativo ad opera dell'art. 42, comma III, del DL n. 269/2003, modificato dalla Legge n. 326/2003, l'unico mezzo di impugnazione è rimasto il ricorso giurisdizionale, che deve essere necessariamente proposto entro il termine perentorio di 6 mesi dalla comunicazione all'interessato, a mezzo raccomandata A.R., del provvedimento emanato in sede amministrativa.

La mancata proposizione del ricorso giurisdizionale entro tale termine decadenziale comporta inevitabilmente l'improcedibilità dell'azione giudiziaria; l'eccezione di decadenza deve essere sollevata, con le modalità e i termini previsti dal rito del lavoro, dalla parte che ne abbia interesse, che deve anche fornire la prova della data di comunicazione all'interessato.

Il ricorso giurisdizionale si propone innanzi al Tribunale in funzione di Giudice del Lavoro nella cui circoscrizione ha residenza il ricorrente, secondo quanto previsto dall'art. 444, comma I, CPC.

Precedentemente all'entrata in vigore del DPR 21 settembre 1994, n. 698, nelle controversie aventi come oggetto l'impugnativa dei provvedimenti definitivi relativi all'accertamento dell'esistenza dei requisiti sanitari e all'erogazione delle provvidenze economiche in tema di prestazioni assistenziali, secondo la Cassazione, la legittimazione passiva competeva al Ministero dell'Interno.

${ }^{8}$ La questione dell'esistenza o meno del diritto degli eredi dell'invalido deceduto nelle more del procedimento amministrativo a percepire le prestazioni economiche maturate a partire dalla presentazione della domanda è stata risolta definitivamente in tal senso dall'art. 1, comma VIII, del DPR n. $698 / 1994$ 
In virtù dell'art. 130 del D. Lgs. 31 marzo 1998, n. 112, la funzione di erogazione di pensioni e indennità è stata trasferita a un apposito Fondo di gestione istituito presso l'INPS; le funzioni di concessione dei nuovi trattamenti economici a favore degli invalidi civili (ad esempio: verifica dei requisiti reddituali, assenza di ricovero, frequenza di un centro riabilitativo o scolastico, ecc) sono invece trasferite alle Regioni, che, secondo il criterio di integrale copertura, provvedono con risorse proprie alla eventuale concessione di benefici aggiuntivi a quelli determinati con legge dello Stato, per tutto il territorio nazionale.

Va anche rilevato che, ai sensi del comma III di detto decreto, «Fermo restando il principio della separazione tra la fase dell'accertamento sanitario e quella della concessione dei benefici economici, di cui all'articolo 11 della legge 24 dicembre 1993, n. 537, nei procedimenti giurisdizionali ed esecutivi, relativi alla concessione delle prestazioni e dei servizi, [...] la legittimazione passiva spetta alle regioni ove il procedimento abbia ad oggetto le provvidenze concesse dalle regioni stesse ed all'INPS negli altri casi».

Ai sensi dell'art. 132, comma II, dello stesso decreto, le Regioni possono conferire ai Comuni e agli altri enti locali le funzioni e i compiti amministrativi concernenti, tra l'altro, i servizi sociali relativi agli invalidi civili.

Successivamente, il DPCM del 26 maggio 2000 stabilisce che dal 01/01/2001 le Regioni devono concretamente esercitare l'attività di concessione delle prestazioni di invalidità civile già svolta dal Ministero dell'Interno.

Dopo il susseguirsi di vari interventi legislativi e provvedimenti giurisprudenziali, dunque, si è delineato un sistema normativo secondo cui la legittimazione passiva appartiene ai seguenti soggetti:

- Ministero dell'Economia e delle Finanze, in qualità di soggetto sovraordinato alla funzione di accertamento dei requisiti sanitari, e quale litisconsorte necessario, ex art. 42 della Legge n. 323/2003;

- INPS, titolare della funzione erogativa delle provvidenze, allorquando la causa abbia come oggetto la concessione delle connesse provvidenze economiche, ai sensi dell'art. 130 del D. Lgs. n. 112/1998. Nei confronti di tale ente lo stato di invalidità deve essere accertato in via incidentale, non essendo necessario promuovere un doppio grado di giudizio [7]:

- Regioni, esclusivamente nei casi di provvidenze aggiuntive dalle stesse previste e finanziate. È dunque da escludersi la legittima- zione passiva delle Regioni sia per le azioni di accertamento e di condanna, sia per quelle di mero accertamento del diritto [8].

Fondamentale importanza riveste poi l'art. 10 della Legge 2 dicembre 2005, n. 248, che stabilisce il trasferimento all'INPS delle funzioni di verifica attribuite in precedenza al Ministero dell'Economia e delle Finanze ${ }^{9}$.

È anche prevista la partecipazione nei ricorsi giurisdizionali in luogo dello stesso Ministero. Infatti, secondo quanto previsto dal comma VI del suddetto articolo: «a decorrere dalla data di effettivo esercizio da parte dell'INPS delle funzioni trasferite, gli atti introduttivi dei procedimenti giurisdizionali in materia di invalidità civile, sordomutismo, handicap e disabilità, nonché le sentenze ed ogni provvedimento reso in detti giudizi, debbano essere notificati anche all'INPS [...]. La notifica va effettuata sia presso gli Uffici dell'Avvocatura dello Stato [...] sia presso le sedi provinciali dell'INPS». Nei summenzionati procedimenti giurisdizionali, infine, l'INPS, è litisconsorte necessario ex art. 102 CPC.

In tal modo, il quadro normativo è stato completamente mutato.

Possiamo, in sintesi, fare la seguente ricognizione:

- le Commissioni mediche di Prima Istanza presso le ASL competenti per territorio assumono la veste funzionale di organi di amministrazione attiva dello Stato, e hanno la specifica funzione di accertare il requisito sanitario con riferimento alle domande amministrative presentate in tutte le materie sopra riportate;

- le Commissioni mediche di Verifica, sia in sede di co-decisione delle prime domande amministrative, sia in sede di verifica successiva del requisito sanitario, esercitano le funzioni statali precedentemente del Ministero dell'Economia e delle Finanze, e il cui concreto esercizio è passato all'INPS;

- ai Comuni di regola è delegata la funzione regionale della concessione della prestazione di assistenza, con il vaglio esclusivo sulla compatibilità del requisito socio-economico;

- I'INPS rimane titolare della funzione di erogazione delle prestazioni.

${ }_{9}$ Il comma I del predetto articolo recita: «L'Istituto Nazionale della Previdenza Sociale (INPS) subentra nell'esercizio delle funzioni residuate allo Stato in materia di invalidità civile, cecità civile, sordomutismo, handicap e disabilità, già di competenza del Ministero dell'Economia e delle Finanze» 


\section{BIBLIOGRAFIA}

1. D. Lgs. n. 112 del 31 marzo 1998 in tema di "Conferimento di funzioni e compiti amministrativi dello Stato alle Regioni ed agli enti locali, in attuazione del capo I della L. 15 marzo 1997, n. 92, S.O."

2. Legge n. 40 del 6 marzo 1998 in tema di "Disciplina dell'immigrazione e norme sulla condizione dello straniero"

3. Legge n. 68 del 12 marzo 1999 in tema di "Norme per il diritto al lavoro dei disabili"

4. Cassazione civile, sez. Lavoro, sentenza n. 5673 del 21 ottobre 1980

5. Consiglio di Stato, sez. VI, sentenza n. 185 del 10 febbraio 1996

6. Cassazione civile, sentenza n. 4641 del 16 aprile 1992

7. Cassazione civile, sentenze n. 6565/2004, n. 15347/2004 e n. 17070/2004

8. Cassazione civile, sentenze n. $15607 / 2004$ e n. $17070 / 2004$

\section{CORRESPONDING AUTHOR}

Avv. Cosimo Prete, e-mail: cosimoprete.avv@libero.it 